\title{
Electromagnetically induced transparency controlled by a microwave field
}

\author{
Hebin Li, ${ }^{1}$ Vladimir A. Sautenkov, ${ }^{1,2}$ Yuri V. Rostovtsev, ${ }^{1}$ George R. Welch, ${ }^{1}$ Philip R. Hemmer, ${ }^{3}$ and Marlan O. Scully ${ }^{1,4}$ \\ ${ }^{1}$ Department of Physics and Institute for Quantum Studies, Texas A\&M University, College Station, Texas 77843-4242, USA \\ ${ }^{2}$ Lebedev Institute of Physics, Moscow 119991, Russia \\ ${ }^{3}$ Electrical Engineering Department, Texas A\&M University, College Station, Texas 77843, USA \\ ${ }^{4}$ Applied Physics and Materials Science Group, Engineering Quad, Princeton University, Princeton, New Jersey 08544, USA
}

(Received 5 March 2009; revised manuscript received 15 May 2009; published 21 August 2009)

\begin{abstract}
We have experimentally studied the propagation of two optical fields in a dense rubidium $(\mathrm{Rb})$ gas in the case when an additional microwave field is coupled to the hyperfine levels of $\mathrm{Rb}$ atoms. The Rb energy levels form a close- $\Lambda$ three-level system coupled to the optical fields and the microwave field. It has been found that the maximum transmission of the probe field depends on the relative phase between the optical and the microwave fields. We have observed both constructive and destructive interferences in electromagnetically induced transparency. A simple theoretical model and a numerical simulation have been developed to explain the observed experimental results.
\end{abstract}

DOI: $10.1103 /$ PhysRevA.80.023820

PACS number(s): 42.50.Gy, 42.25.Kb, 33.20.Bx

\section{INTRODUCTION}

Electromagnetically induced transparency (EIT) is based on quantum coherence [1-5] that has been shown to result in many counterintuitive phenomena. The scattering via a gradient force in gases [6], the forward Brillouin scattering in ultradispersive resonant media [7-9], the controlled coherent multiwave mixing $[10,11]$, the EIT, and the slow light in various media [12-18], the Doppler broadening elimination [19], the light-induced chirality in a nonchiral medium [20], the switching faster than the relaxation rate [21], a new class of entanglement amplifier [22] based on correlated spontaneous emission lasers [23,24], and the coherent Raman scattering enhancement via maximal coherence in atoms [25,26] and biomolecules [27-30] are a few examples that demonstrate the importance of quantum coherence.

Usually, the EIT has been observed in atoms that have a three-level configuration such as $\Lambda, \mathrm{V}$, and ladder schemes $[1,2]$. For these schemes several theoretical approaches have been developed to provide clear physical insights. For example, the EIT can be understood in the bare state basis using quantum coherence, or in the dressed state basis involving Fano interference, or using the so-called dark and bright states [1,2].

Natural generalization of the three-level schemes is the so-called double- $\Lambda$, double- $\mathrm{V}$, double-ladder, and $\Lambda-\mathrm{V}$ schemes [31], where two relatively strong optical fields are applied to the atomic system to create coherence, and then a probe field propagates through the gas of such atoms together with an additional strong drive field. The probe propagation depends on the parameters of the medium and the fields preparing coherence. For instance, the relative phase of optical fields is crucial to determine the optical response of the medium in a $\Lambda-\mathrm{V}$ scheme [32]. On the other hand, let us note that the effect of these two optical drive fields is equivalent to an effective microwave drive field applied to the system. Furthermore, in some regard, the schemes that involve two optical fields and a microwave field can be related to the double- $\Lambda$ scheme.

The systems involving interaction with two optical fields and a low-frequency microwave field coupled to the hyper- fine levels have been in a focus of recent studies [33]. For example, microwave interaction [34] has been used to excite the Raman trapped state and it was shown that there is an influence of the microwave field on the coherent population trapping in a $\Lambda$ system; four-wave mixing of optical and microwave fields has been demonstrated [35] in a $\mathrm{Rb}$ gas. A microwave field has also been used to study double-dark resonances [36]. It has been shown [37] that, in a V-scheme three-level system of $\mathrm{Pr}^{3+}: \mathrm{YAlO}_{3}$ that was excited by a microwave drive field and two optical probe fields, the probe transmission was either constructively or destructively affected by the phase of the microwave field.

Recently, the phase effects in EIT systems has been studied [38-40], where the transient times of the refractive Kerr nonlinearity have been studied and it has been shown that the refractive Kerr nonlinearity is enhanced using EIT. Besides, these close systems also have broad range of applications that stimulated our interest to this system. For example, they have been considered as perspective candidates for realization of stop-and-go slow light $[41,42]$ and backward scattering $[10,11]$. Furthermore, the interest to this topic is stimulated by a recent work [43], in which a quantum storage based on electromagnetically induced transparency has been predicted. The first experiments in support of the theoretical predictions have also been performed [43]. In [44], it was shown that the quantum state of light can be stored and retrieved in a dense medium by switching on and off a control field. A quantum state of light having one polarization and carrier frequency can be transferred to the same state of light but having a different carrier frequency, polarization, or direction of propagation. These systems with a microwave field have a better controlled probe transparency because the absorption of the microwave field is much smaller than optical fields, which is important for improving and optimizing quantum storage efficiency $[45,46]$. Slow light produces delay that can be used in optical buffers; the delay time is limited by the absorption of probe field. Using an auxiliary microwave field can improve the important parameter for broadband systems, the product of delay time and bandwidth of the pulse [47], which shows the effective number of communication channels. 


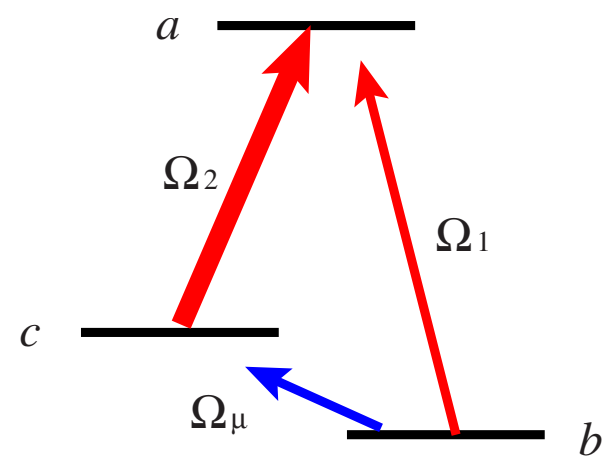

FIG. 1. (Color online) Energy levels of a closed $\Lambda$ scheme three-level system. $\Omega_{1}$ and $\Omega_{2}$ are two optical fields and $\Omega_{\mu}$ is a microwave field.

In this paper, we report on the study of EIT in a threelevel $\Lambda$ scheme interacting with two optical fields and a microwave field coupled with hyperfine levels. "Perturbing" (due to a microwave field) the coherence of two ground states leads the change in maximum transmission of probe field. Either enhanced or suppressed EIT peaks can be obtained depending on the relative phase between the optical fields and the microwave field. The paper is organized by starting with a simple theoretical model of a close- $\Lambda$ scheme. Then, we describe experimental details and obtained results. At the end we present a numerical simulation that reproduces experimental results.

\section{THEORY}

Let us consider a cell filled with a gas of three-level atoms that have the energy level scheme shown in Fig. 1. Two optical beams are propagating through the cell of atoms along $z$ axis, and the optical fields

$$
E_{1}=\mathcal{E}_{1} \cos \left(\nu_{1} t-k_{1} z+\phi_{1}\right)
$$

and

$$
E_{2}=\mathcal{E}_{2} \cos \left(\nu_{2} t-k_{2} z+\phi_{2}\right)
$$

are coupled to the atomic transitions $|a\rangle \rightarrow|b\rangle$ and $|a\rangle \rightarrow|c\rangle$ (see Fig. 1) correspondingly, where $\nu_{1}$ and $\nu_{2}$ are the optical frequencies of the corresponding fields; $k_{1}$ and $k_{2}$ are the wave numbers of the optical fields; and $\phi_{1}$ and $\phi_{2}$ are the phases of the optical fields. The cell is placed in a microwave cavity and a microwave field

$$
E_{\mu}=\mathcal{E}_{\mu}(z) \cos \left(\nu_{\mu} t+\phi_{\mu}\right)
$$

is coupled to two ground states $|b\rangle$ and $|c\rangle$. Here $\mathcal{E}_{\mu}(z)$ is the dependence of the microwave field on position $z$ inside the cavity, $\nu_{\mu}$ is the microwave frequency, and $\phi_{\mu}$ is the phase of the microwave field.

The Hamiltonian of the atom located at position $z$ can be written as

$$
\begin{aligned}
H= & \hbar \omega_{a}|a\rangle\left\langle a\left|+\hbar \omega_{b}\right| b\right\rangle\left\langle b\left|+\hbar \omega_{c}\right| c\right\rangle\langle c|-\left[\wp_{a b} E_{1}|a\rangle\langle b|\right. \\
& \left.+\wp_{a c} E_{2}|a\rangle\left\langle c\left|+\wp_{b c} E_{\mu}\right| c\right\rangle\langle b|+\text { H.c. }\right],
\end{aligned}
$$

where $\hbar \omega_{\alpha}$ is the energy of the corresponding level $|\alpha\rangle$ and $\wp_{\alpha, \beta}=\langle\alpha|\hat{\wp}| \beta\rangle$ are the dipole moments of corresponding transitions $|\alpha\rangle \rightarrow|\beta\rangle(\alpha, \beta=a, b, c)$.

The density-matrix equation of motion [2] is given by

$$
\dot{\rho}=-\frac{i}{\hbar}[H, \rho]-\frac{1}{2}\{\Gamma, \rho\},
$$

where $\{\Gamma, \rho\}=\Gamma \rho+\rho \Gamma$ and $\Gamma$ is the relaxation matrix. The nondiagonal elements of the density-matrix equations are found as follows:

$$
\begin{aligned}
\dot{\rho}_{a b}= & -\left(\gamma_{a b}+i \omega_{a b}\right) \rho_{a b}-i \frac{\wp_{a b} E_{1}}{\hbar}\left(\rho_{a a}-\rho_{b b}\right)+i \frac{\wp_{a c} E_{2}}{\hbar} \rho_{c b} \\
& -i \frac{\wp_{c b} E_{\mu}}{\hbar} \rho_{a c}, \\
\dot{\rho}_{a c}= & -\left(\gamma_{a c}+i \omega_{a c}\right) \rho_{a c}-i \frac{\wp_{a c} E_{2}}{\hbar}\left(\rho_{a a}-\rho_{c c}\right)+i \wp_{a b} E_{1} \rho_{b c} \\
& -i \frac{\wp_{b c} E_{\mu}}{\hbar} \rho_{a b}, \\
\dot{\rho}_{c b}= & -\left(\gamma_{c b}+i \omega_{c b}\right) \rho_{c b}-i \frac{\wp_{c b} E_{\mu}}{\hbar}\left(\rho_{c c}-\rho_{b b}\right)+i \frac{\wp_{c a} E_{2}}{\hbar} \rho_{a b} \\
& -i \frac{\wp_{a b} E_{1} \rho_{c a}}{\hbar},
\end{aligned}
$$

where $\omega_{\alpha \beta}=\omega_{\alpha}-\omega_{\beta}$.

At this point, we make the rotating wave approximation by leaving only the resonance terms and neglecting the counter-rotating far-off resonance terms. Introducing slowly varying envelopes $\rho_{a b}=\sigma_{a b} \exp \left(i k_{1} z-i \nu_{1} t\right), \quad \rho_{a c}$ $=\sigma_{a c} \exp \left(i k_{2} z-i \nu_{2} t\right), \quad \rho_{c b}=\sigma_{c b} \exp \left[i \Delta k z-i\left(\nu_{1}-\nu_{2}\right) t\right], \quad$ and $\sigma_{\alpha \alpha}=\rho_{\alpha \alpha}$, where $\Delta k=k_{1}-k_{2}$, then the density-matrix equations can be written as

$$
\begin{gathered}
\dot{\sigma}_{a b}=-\Gamma_{a b} \sigma_{a b}-i \Omega_{1}\left(\sigma_{a a}-\sigma_{b b}\right)+i \Omega_{2} \sigma_{c b} \\
-i \Omega_{\mu} e^{i\left(\nu_{1}-\nu_{2}-\nu_{\mu}\right) t+i \Delta k z} \sigma_{a c}, \\
\dot{\sigma}_{a c}=-\Gamma_{a c} \sigma_{a c}-i \Omega_{2}\left(\sigma_{a a}-\sigma_{c c}\right)+i \Omega_{1} \sigma_{b c} \\
-i \Omega_{\mu}^{*} e^{-i\left(\nu_{1}-\nu_{2}-\nu_{\mu}\right) t-i \Delta k z} \sigma_{a b}, \\
\dot{\sigma}_{c b}=-\Gamma_{c b} \sigma_{c b}-i \Omega_{\mu} e^{i\left(\nu_{1}-\nu_{2}-\nu_{\mu}\right) t+i \Delta k z}\left(\sigma_{c c}-\sigma_{b b}\right)+i \Omega_{2}^{*} \sigma_{a b} \\
-i \Omega_{1} \sigma_{c a},
\end{gathered}
$$

where

$$
\Omega_{1}=\frac{\wp_{a b} \mathcal{E}_{1}}{2 \hbar} e^{i \phi_{1}}, \quad \Omega_{2}=\frac{\wp_{a c} \mathcal{E}_{2}}{2 \hbar} e^{i \phi_{2}}, \quad \Omega_{\mu}=\frac{\wp_{b c} \mathcal{E}_{\mu}}{2 \hbar} e^{i \phi_{\mu}}
$$

are the Rabi frequencies of the optical probe field, the optical drive field, and the microwave field, respectively; $\Gamma_{a b}=\gamma_{a b}$ $+i\left(\omega_{a b}-\nu_{1}\right) ; \quad \Gamma_{a c}=\gamma_{a c}+i\left(\omega_{a c}-\nu_{2}\right) ;$ and $\Gamma_{c b}=\gamma_{c b}+i\left(\omega_{c b}+\nu_{2}\right.$ $\left.-\nu_{1}\right)$. We consider the case in which the drive field is on resonant $\left(\nu_{2}=\omega_{a c}\right)$, while the probe field and the microwave field have the same detuning $\Delta \equiv \omega_{a b}-\nu_{1}=\omega_{c b}-\nu_{\mu}$; thus $\nu_{1}$ $-\nu_{2}-\nu_{\mu}=0$. We consider the steady-state regime $\left(\dot{\rho}_{a b}=\dot{\rho}_{a b}\right.$ $=\dot{\rho}_{c b}=0$ ) and assume that the drive field is much stronger 
than the probe field and the microwave field $\left(\left|\Omega_{2}\right|\right.$ $\left.\gg\left|\Omega_{1}\right|,\left|\Omega_{\mu}\right|\right)$, so all of the population is in the ground state $|b\rangle$, i.e., $\rho_{b b} \simeq 1$ and $\rho_{a a}=\rho_{c c} \simeq 0$; we can solve Eqs. (6)-(8) for $\sigma_{a b}$,

$$
\sigma_{a b}=\frac{i \Gamma_{c b} \Omega_{1}}{\Gamma_{a b} \Gamma_{c b}+\left|\Omega_{2}\right|^{2}}-\frac{\Omega_{2} \Omega_{\mu} e^{i \Delta k z}}{\Gamma_{a b} \Gamma_{c b}+\left|\Omega_{2}\right|^{2}}
$$

with $\Gamma_{a b}=\gamma_{a b}+i \Delta$ and $\Gamma_{c b}=\gamma_{c b}+i \Delta$.

The propagation of the probe field is described by the Maxwell equation [2]

$$
\frac{\partial^{2} E_{1}}{\partial z^{2}}-\frac{1}{c^{2}} \frac{\partial^{2} E_{1}}{\partial t^{2}}=-\frac{\nu_{1}^{2}}{c^{2} \epsilon_{0}} N\left(\wp_{a b} \sigma_{a b}+\text { c.c. }\right),
$$

where $N$ is the atomic density and $\epsilon_{0}$ is the permittivity in vacuum. Then, we obtain the propagation equation for the probe field in the slowly varying amplitude approximation as

$$
\frac{\partial \Omega_{1}}{\partial z}=-i \eta \sigma_{a b},
$$

where $\eta=\nu_{1} N \wp_{a b}^{2} /\left(2 \epsilon_{0} c \hbar\right)$ is the coupling constant.

We consider this point until the case of the atom at rest. To take into account the motion of atoms, we introduce kinetic-energy term in the Hamiltonian (see details in Appen$\operatorname{dix}$ A). The final result looks like as if we assume that the atom is moving with some velocity $v$, then the $\Gamma$ 's should include the Doppler shifts due to the atomic motion. Namely, they should be defined as

$$
\begin{gathered}
\Gamma_{a b}=\gamma_{a b}+i\left(k_{1} v+\Delta\right), \\
\Gamma_{a c}=\gamma_{a c}+i k_{2} v, \\
\Gamma_{c b}=\gamma_{c b}+i\left[\Delta+\left(k_{1}-k_{2}\right) v\right],
\end{gathered}
$$

and the optical atomic polarization should be substituted by the averaged over the atomic distribution on velocities as

$$
\left\langle\sigma_{a b}\right\rangle_{v}=\frac{1}{\sqrt{\pi u_{D}^{2}}} \int_{-\infty}^{+\infty} d v \sigma_{a b}(v) \exp \left(-\frac{v^{2}}{u_{D}^{2}}\right),
$$

where $u_{D}$ is the most probable speed of atoms. As is well known, the one-photon transitions are broadened due to the Doppler broadening. The broadening of the probe transition is on the order of $k_{1} u_{D}$; meanwhile, the broadening of the two-photon transition depends on the geometry of the drive and the probe fields. The broadening is different for copropagating and contrapropagating fields. For our case, the broadening of the two-photon transition is on the order of $\left(k_{1}\right.$ $\left.-k_{2}\right) u_{D} \simeq 7.5 \mathrm{kHz}$ (besides, the collisions with the buffer gas suppress the two-photon Doppler broadening due to the Dicke effect) that is comparable with the relaxation at the hyperfine transition $\gamma_{c b}$. One can see that the power broadening under experimental condition is broader than the twophoton Doppler broadening. As has been shown in [48], the effect of the Doppler broadening at one-photon transition can be taken into account by incorporating the Doppler width of optical transitions, $k_{1} u_{D}$ and $k_{2} u_{D}$, in $\Gamma_{a b}=\gamma_{a b}+k_{1} u_{D}+i \Delta$ and $\Gamma_{a c}=\gamma_{a c}+k_{2} u_{D}$ and neglecting the Doppler width of two- photon transition. Using this approach, we have taken into account the Doppler broadening.

Thus, combining Eqs. (9) and (11), we obtain the propagation equation

$$
\frac{\partial \Omega_{1}}{\partial z}=-\frac{\eta \Gamma_{c b} \Omega_{1}}{\Gamma_{a b} \Gamma_{c b}+\left|\Omega_{2}\right|^{2}}-i \frac{\eta \Omega_{2} \Omega_{\mu} e^{i \Delta k z}}{\Gamma_{a b} \Gamma_{c b}+\left|\Omega_{2}\right|^{2}} .
$$

On the right-hand side of Eq. (16), the first term is due to the contribution from the $\Lambda$-scheme EIT, and the second term is the contribution from the parametric process involved the microwave field. The transmission of the probe field is determined by the interference of these two terms. The second term is interesting because of the strong dependence on the relative phase of the optical fields and the microwave field. This gives us several ways to control the coherence and the transmission of the probe field. For instance, one can use a microwave phase shifter to change the phase of microwave field; one can also use an optical delay line, like the one used in [34], to change the phase of optical field. An alternative way is simply changing the position of the $\mathrm{Rb}$ cell, which is described as the following.

Assume that the drive and the probe fields are phase locked; they form a wave package along the propagation direction with the frequency, which is the frequency difference of two fields. For ${ }^{87} \mathrm{Rb}$, this frequency is $6.835 \mathrm{GHz}$, and the corresponding wavelength is about $4.4 \mathrm{~cm}$. If we put the $\mathrm{Rb}$ cell in a microwave cavity, which is excited by a microwave with the frequency $6.835 \mathrm{GHz}$, the phase of microwave field in the cavity does not change when we move the cell and the microwave cavity together. However, the relative optical phase changes since the relative position of the cell with respect to the wave package of optical fields changes. In other words, we are able to change the phase $\Delta k z$ by moving the cell and the microwave cavity along the propagation direction of the optical fields.

\section{EXPERIMENT}

\section{A. Experimental setup}

The experimental setup is schematically shown in Fig. 2. The ${ }^{87} \mathrm{Rb}$ gas along with 5 Torr of neon buffer gas is confined in a glass cell with the length of $25 \mathrm{~mm}$. To efficiently couple the microwave field with $\mathrm{Rb}$ atoms, the $\mathrm{Rb}$ cell is installed in a microwave cavity. The microwave cavity with the cell is installed in a magnetic shield to isolate the cell from environmental magnetic fields in the laboratory, and the residue magnetic field is negligible. The Zeeman sublevels are not resolved in our experiment. A nonmagnetic heater is used to heat the cell to reach the atomic density of $10^{12} \mathrm{~cm}^{-3}$. The microwave cavity has the resonant frequency of $6.835 \mathrm{GHz}$. The loaded quality factor $Q$ is about 2000, and the cavity mode is $\mathrm{TE}_{011}$. A microwave signal generator provides the $6.835 \mathrm{GHz}$ microwave field, which is amplified by a microwave amplifier and is then injected into the cavity through an antenna. The $6.835 \mathrm{GHz}$ microwave field is coupled with two hyperfine ground states $5 S_{1 / 2}(F=1)$ and $5 S_{1 / 2}(F=2)$ of ${ }^{87} \mathrm{Rb}$ atoms.

The optical fields are provided by a diode laser, which is tuned to the $D_{1}$ resonance line of ${ }^{87} \mathrm{Rb}$ atoms, specifically at 


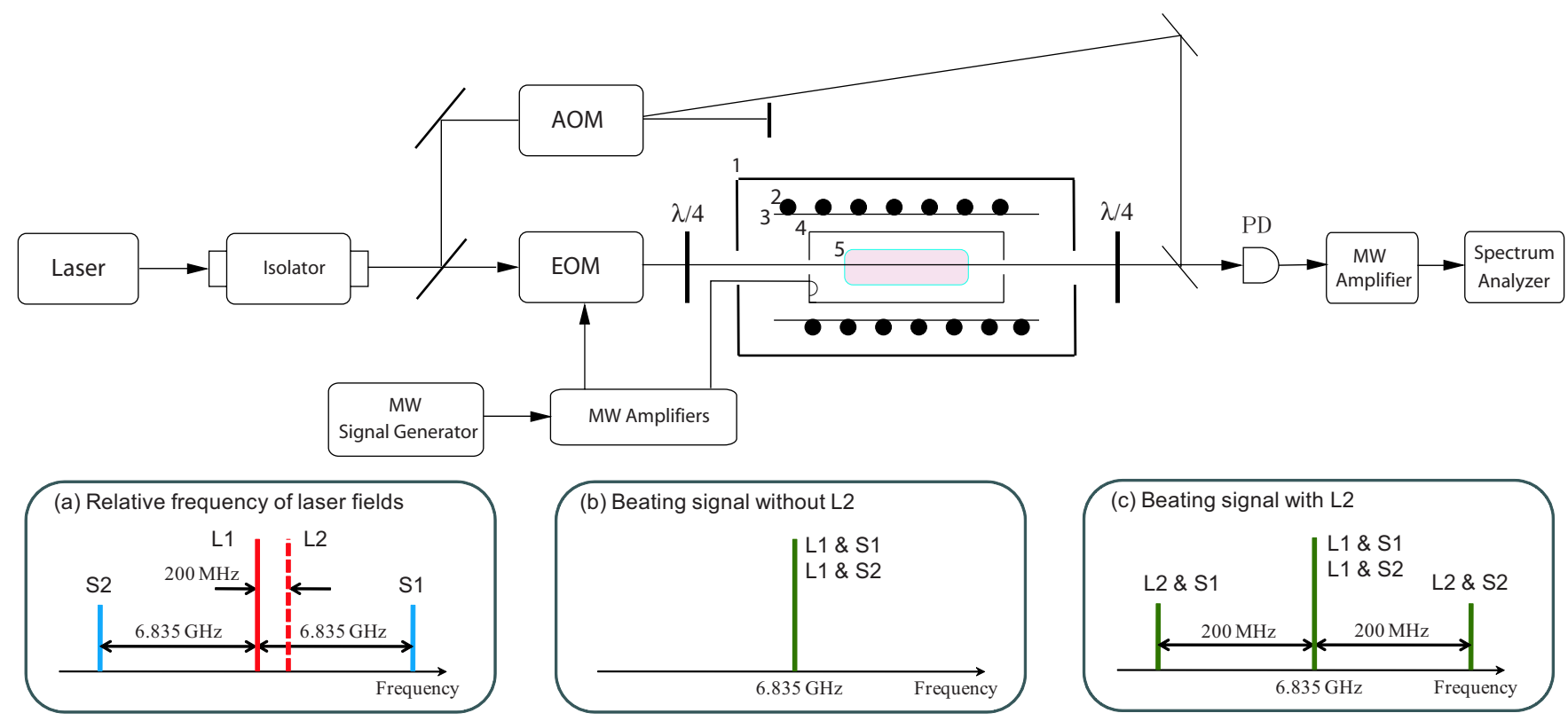

FIG. 2. (Color online) Experimental setup. EOM, electro-optic modulator; AOM, acousto-optic modulator; PD, photodiode; the oven is assembled with 1, copper tube; 2, nonmagnetic heater; 3, magnetic shield; 4, microwave cavity with antenna; 5 , Rb cell. Inset (a) shows the relative frequency of the laser fields. L1 is the drive field, S1 and S2 are two sidebands generated by the EOM and S1 works as the probe field. L2 is the laser field shifted $200 \mathrm{MHz}$ in frequency by the AOM. Inset (b) shows the beating signal without considering L2. Inset (c) shows the beating signals with all fields L1, L2, S1, and S2 considered.

the transition $5 S_{1 / 2}(F=2) \leftrightarrow 5 P_{1 / 2}(F=2)$. The laser beam is split into two beams by a beam splitter. One beam passes through an acousto-optic modulator (AOM) which shifts the laser frequency by $200 \mathrm{MHz}$. This beam with $200 \mathrm{MHz}$ shift in frequency is denoted as L2 in Fig. 2(a). It will be used as a local oscillator of the heterodyne detection. The other beam is used to make the drive and the probe fields. This beam passes through an electro-optic modulator (EOM) which is driven by a microwave field with the frequency of 6.835 GHz. Two sidebands are generated with the frequency separation of $6.835 \mathrm{GHz}$ with respect to the original field. Two sidebands are denoted as S1 and S2 in Fig. 2(a). The original field works as the drive field. One sideband (S1) works as the probe field at the transition $5 S_{1 / 2}(F=1) \leftrightarrow 5 P_{1 / 2}(F=2)$. The intensity ratio of the probe field to the drive field is about $15 \%$. The other sideband (S2) is $6.835 \mathrm{GHz}$ downshifted in frequency with respect to the drive field. It is far detuned from the resonance and it has a negligible effect on the experiment. The laser beam from the EOM is circularly polarized by a quarter wave plate and it is directed into the $\mathrm{Rb}$ cell.

With the optical fields (drive and probe) coming out from the EOM and the microwave field in the cavity, we have a closed- $\Lambda$ system as shown in Fig. 1 . Note that the microwave signals used by the EOM and the microwave cavity come from the same microwave signal generator. During the experiment, the microwave generator is modulated by $200 \mathrm{kHz}$ in frequency around $6.835 \mathrm{GHz}$ and the sweep period is 0.1 $\mathrm{s}$. Therefore, the probe laser field and the microwave field are synchronized to scan $200 \mathrm{kHz}$ in frequency. In other words, they have the same detuning.

The transmitted probe field is detected by the heterodyne detection used in Ref. [13]. Instead of a direct measurement of the transmitted intensity, which contains the drive and the two sidebands, we detect the beating signal using a fast photodetector with the bandwidth of $25 \mathrm{GHz}$. The signal from the photodetector is analyzed by a spectrum analyzer, which is synchronized with the modulation of the microwave generator. As shown in Fig. 2(b), without the presence of the shifted drive beam L2, the detected beating signal is at 6.835 $\mathrm{GHz}$. It includes the beating signal between L1 and S1 and the beating signal between L1 and S2. With the frequency shifted drive beam L2, it beats with the transmitted beam to separate the frequency components of S1 and S2 shown in Fig. 2(c). The beating signal between L2 and S2 is blueshifted by $200 \mathrm{MHz}$, and the beating signal between L2 and $\mathrm{S} 1$ is redshifted by $200 \mathrm{MHz}$. The transmission of the probe field is obtained by measuring the beating signal at the frequency of $6.835 \mathrm{GHz}+200 \mathrm{MHz}$.

\section{B. Experimental results}

We start the presentation of experimental results by showing the transmission of the probe field as a function of the detuning. We plot the transmission versus the detuning in Fig. 3. A normal EIT transmission peak without applying the microwave field is shown in Fig. 3(b). With the presence of the microwave field, we expect that the ground-state coherence is "disturbed" by the microwave field and it changes the transmission of the probe field. As discussed above, we change the relative phase between the optical fields and the microwave field by changing the position of the cell and the microwave cavity along the optical axis. The periodicity of the cell position influencing the probe field transmission is expected to be about $4.4 \mathrm{~cm}$, which is the wavelength of the microwave field with the frequency of $6.835 \mathrm{GHz}$. Due to 


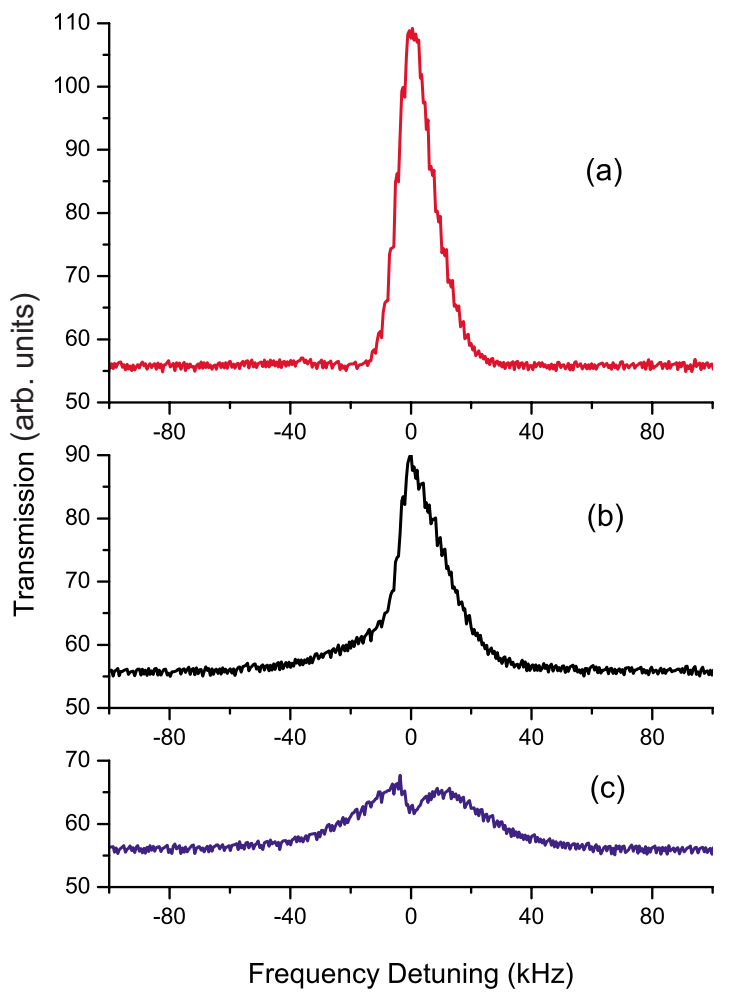

FIG. 3. (Color online) The beating signal recorded by a spectrum analyzer at the center frequency of $7.035 \mathrm{GHz}$. It represents the transmission of the probe field. (a) The enhanced transmission with the microwave field applied; (b) the transmission without the microwave field applied; (c) the suppressed transmission with the microwave field applied. The positions of the cell where (a) and (c) were taken are about $2.2 \mathrm{~cm}$ apart.

the interference of two terms on the right-hand side of Eq. (9), the transmission of probe field could be either enhanced or suppressed depending on the relative phase. It is what we have observed in the experiment. As shown in Fig. 3, with applying the microwave field, we have obtained both enhanced [Fig. 3(a)] and suppressed [Fig. 3(c)] transmissions of the probe field as we move the microwave cavity with the cell to change the relative phase between the optical fields and the microwave field. The positions of the cell corresponding to curves (a) and (c) are at the distance of about 2.2 $\mathrm{cm}$.

An interesting feature needs to be pointed out for the case of suppressed transmission [Fig. 3(c)]. In this case, the amplitude of the EIT peak decreases as we expected, and we also have a small dip on the top, which indicates that one (the one due to presence of microwave field) of the interfering terms has a relatively narrower width. Its width is narrower than the EIT width.

To systematically study the influence of the relative phase between the optical fields and the microwave field on the probe field transmission, the EIT transmission is recorded at different positions of the cell with a step of $3 \mathrm{~mm}$ along the propagation direction of the optical fields as shown in Fig. 4. The transmission has been obtained with different polarizations of optical fields. The results obtained with the right circularly polarized laser field are shown in Fig. 4(a), and the results with the left circularly polarized laser field are shown in Fig. 4(b). The amplitude of the EIT peak is oscillating with the change in the cell position. The distance between two maxima (or minima) next to each other is about $4.4 \mathrm{~cm}$, which is exactly the wavelength of the beating envelope of the input optical fields. This periodicity is consistent with the theoretical prediction described above.

The oscillation is shown in Fig. 5, where we plot the amplitude of EIT peaks as a function of the relative phase (phase $2 \pi$ corresponds to the wavelength of $4.4 \mathrm{~cm}$ ). The dash lines are fittings of the sinusoidal function. Comparing the cases of right and left circularly polarized input laser fields, the behaviors are exactly opposite. This feature seems to be surprising, because the whole system is symmetrical about the optical axis and there is no obvious way to tell the difference between left and right circular polarizations. The physics of such behavior is the following. The left and the right circularly polarized fields are coupled with different Zeeman sublevels (see Fig. 6). Due to the optical pumping, among the Zeeman sublevels of the ground state with $F=1$,
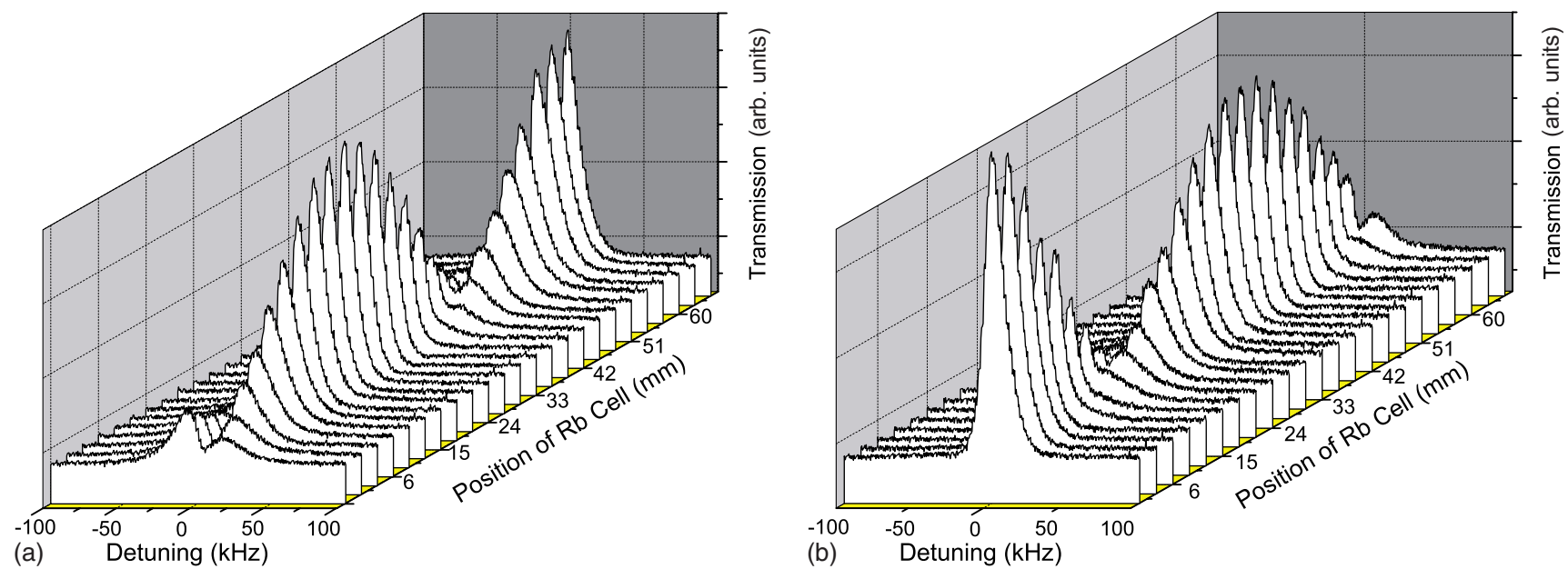

FIG. 4. (Color online) The EIT peaks as we change the position of the cell along the propagation direction of optical fields. An EIT peak is recorded at every $3 \mathrm{~mm}$ we move the cell. (a) and (b) correspond to the case where the input laser fields are right and left, respectively, circularly polarized. The distance between two maxima (or minima) next to each other is about $4.4 \mathrm{~cm}$. 


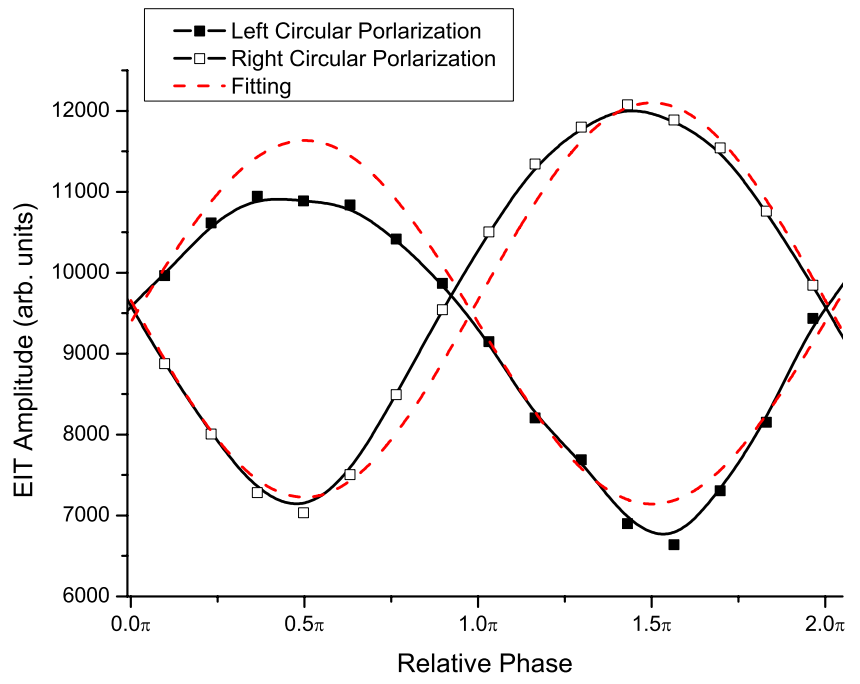

FIG. 5. (Color online) The amplitude of EIT peaks dependence on relative phase. The solid and hollow squares correspond to the cases of left and right circularly polarized input laser fields, respectively. Dash lines are fittings of the sinusoidal function.

the state $|F=1, m=+1\rangle$ is most populated for the right circularly polarized light and the state $|F=1, m=-1\rangle$ is most populated for the left circularly polarized light. Then, we can see a simplified level scheme shown in Fig. 1 corresponding to the real $\mathrm{Rb}$ energy levels. For the right circularly polarized fields, we have $|a\rangle=\left|F^{\prime}=2, m=2\right\rangle,|b\rangle=|F=1, m=1\rangle$, and $|c\rangle=|F=2, m=1\rangle$. For the left circularly polarized fields, we have $|a\rangle=\left|F^{\prime}=2, m=-2\right\rangle,|b\rangle=|F=1, m=-1\rangle$, and $|c\rangle$ $=|F=2, m=-1\rangle$. The magnetic transition dipole moments between hyperfine levels are equal to each other as

$$
\left\langle 2,-m\left|\hat{\mu}_{z}\right| 1,-m\right\rangle=\left\langle 2, m\left|\hat{\mu}_{z}\right| 1, m\right\rangle,
$$

while the relation between the dipole moments of optical transitions (see Appendix B) are the following:

$$
\begin{gathered}
\left\langle 2^{\prime}, m+1\left|\wp_{+1}\right| 2, m\right\rangle=-\left\langle 2^{\prime},-m-1\left|\wp_{-1}\right| 2,-m\right\rangle, \\
\left\langle 2^{\prime}, m+1\left|\wp_{+1}\right| 2, m\right\rangle=\left\langle 2^{\prime},-m-1\left|\wp_{-1}\right| 1,-m\right\rangle,
\end{gathered}
$$

where we use the notations $\left|2^{\prime}, m\right\rangle=\left|F^{\prime}=2, m\right\rangle$ and $|2, m\rangle$ $=|F=2, m\rangle$. Consequently, for the simplified model (shown in Fig. 1), the Rabi frequencies for the right and the left

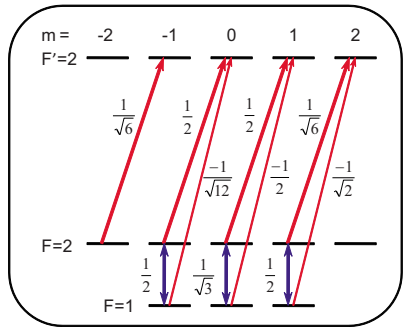

(a)

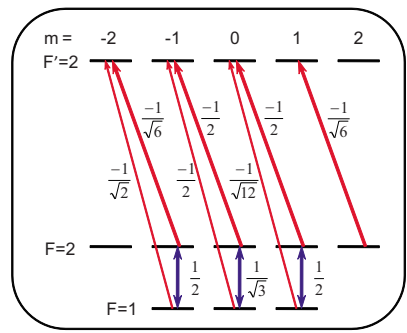

(b)
FIG. 6. (Color online) Rb level scheme. The coupling between (a) the right and (b) the left circularly polarized optical probe and drive beams and $\mathrm{Rb}$ levels. Near each transition, the corresponding Clebsch-Gordan factors (see Appendix B) are shown.

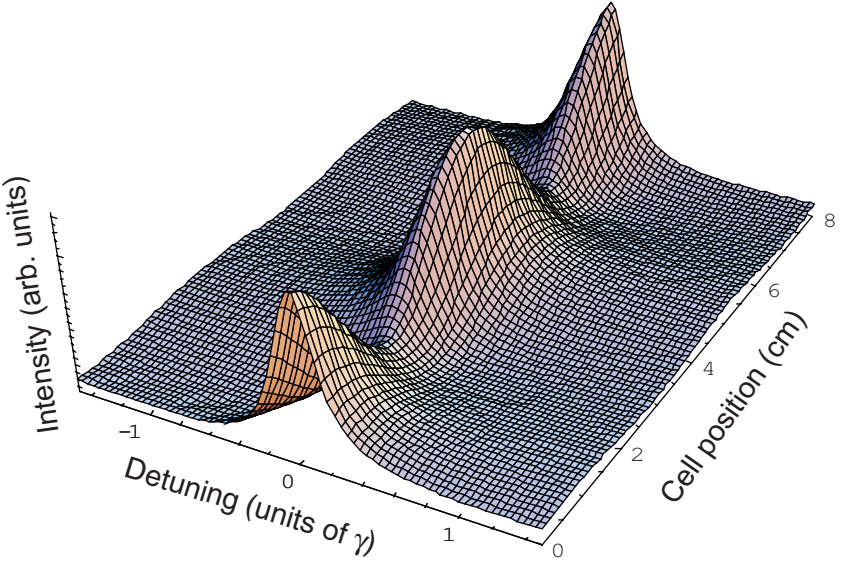

FIG. 7. (Color online) Numerical simulation of the transmission of the probe field dependence on detuning and cell positions. In the simulation, we use $\gamma_{a b}=5, \gamma_{b c}=10^{-3}, \Omega_{10}=0.1, \Omega_{2}=1, \Omega_{\mu}=0.02$, $\eta=0.9, L=2.5 \mathrm{~cm}$, and $\Delta k=1.5 \mathrm{~cm}^{-1}$.

circularly polarized optical fields have the following relations:

$$
\Omega_{1}^{r}=\Omega_{1}^{l}, \quad \Omega_{2}^{r}=-\Omega_{2}^{l},
$$

where the indices $r$ and $l$ denote the Rabi frequencies of the right and the left circularly polarized optical beams, respectively. Thus, the corresponding change in the phase of the Rabi frequencies of the optical beams has opposite signs, which introduce a phase difference of $\pi$ in our results.

\section{SIMULATION}

We perform a simulation based on Eq. (16). Assume that the length of the Rb cell be $L$, and the optical fields enter the $\mathrm{Rb}$ cell at position $z_{0}$ and leave at position $z_{0}+L$. With the probe field $\Omega_{10}$ entering the cell, Eq. (16) gives the transmitted probe field $\Omega_{1}$ as follows:

$$
\begin{aligned}
\Omega_{1}\left(z_{0}+L\right)= & \Omega_{10} e^{-\alpha L}-i \frac{\eta \Omega_{\mu} \Omega_{2}}{\Gamma_{c b} \Gamma_{a b}+\left|\Omega_{2}\right|^{2}} \frac{1}{i \Delta k+\alpha}\left[e^{i \Delta k\left(z_{0}+L\right)}\right. \\
& \left.-e^{i \Delta k z_{0}-\alpha L}\right],
\end{aligned}
$$

where $\alpha$ is the absorption coefficient, which is given by

$$
\alpha=\eta \frac{\Gamma_{c b}}{\Gamma_{c b} \Gamma_{a b}+\left|\Omega_{2}\right|^{2}} .
$$

The simulation result is shown in Fig. 7. The parameters we used in the simulation are the following: $\gamma_{a b}=5, \gamma_{b c}$ $=10^{-3}, \Omega_{10}=0.1, \Omega_{2}=1, \Omega_{\mu}=0.02, \eta=0.9, L=2.5 \mathrm{~cm}$, and $\Delta k=1.5 \mathrm{~cm}^{-1}$. As varying the detuning, the maximum transmission appears at zero detuning. Meanwhile, the maximum transmission is oscillating when we change $z_{0}$, which determines the position of the $\mathrm{Rb}$ cell, and the period of oscillation is about $4.4 \mathrm{~cm}$. The simulation shows the similar behavior as the experimental results.

It is interesting to note that the obtained results can be considered for the realization of the stop-and-go slow light $[41,42]$. The dispersion can be modified in a controllable 
way that slows or accelerates the group velocity of light by tuning the frequency of the microwave field near the resonance with the hyperfine transition.

The obtained results can be also applied to the backward scattering predicted in $[10,11]$. By controlling the dispersion of the medium with the optical fields, a microwave field can be produced. Its direction of propagation is determined by the parameters of the fields, in particular, the detuning of the optical fields from the two-photon resonance.

Furthermore, the interest in this topic is stimulated by the recent work [43] in which a quantum storage based on electromagnetically induced transparency has been predicted. The delay produced by slow light can be used in optical buffers; the delay time is limited by the absorption of probe field. Because the absorption of a microwave field is much smaller than optical fields, these systems have a better controlled probe transparency, which is important for improving and optimizing the efficiency of quantum storage $[45,46]$. Using auxiliary microwave field can improve the product of delay time and the bandwidth for the pulse case [47]. The broad range of applications stimulated our interest in the atomic system with the optical and the microwave fields.

\section{CONCLUSION}

In conclusion, we have experimentally studied EIT in Rb atoms coupled with two optical fields and a microwave field. The microwave is coupled to two hyperfine levels, and coherently perturbs the coherence of two hyperfine levels, thus changing the transmission of probe field. It has been found that the maximum transmission of probe field depends on the relative phase between the optical fields and the microwave field, and both enhanced and suppressed EIT peaks have been observed. A simple theoretical model and a numerical simulation have been provided. The simulation shows the similar behavior as the experimental results.

\section{ACKNOWLEDGMENTS}

We thank D. X. Ma, A. B. Matsko, C. Shin, A. V. Sokolov, and A. S. Zibrov for useful and fruitful discussions. This work was supported by the Welch Foundation (Grant No. A-1261), the NSF Grant No. EEC-0540832 (MIRTHE ERC), and the Office of Naval Research (Grants No. N00014-07-11084 and No. N0001408-1-0948).

\section{APPENDIX A: TAKING INTO ACCOUNT DOPPLER BROADENING}

Even though the Doppler broadening is a common effect in atomic and molecular spectroscopy [49], here we briefly sketch a regular approach as well as two simplified approaches used in the literature [50]. To take into account the motion of atoms, we introduce an operator of kinetic energy in the Hamiltonian that describes the center-of-mass motion. Consider, for simplicity, a three-level atom that can move in only one direction. It has the Hamiltonian given by

$$
\hat{H}=\frac{\hat{p}^{2}}{2 M}+\hbar \omega_{a b}|a\rangle\left\langle a\left|+\hbar \omega_{c b}\right| c\right\rangle\langle c|+\hat{V},
$$

where $M$ is the mass of the atom and

$$
\hat{V}=\wp_{1} E_{1}|a\rangle\left\langle b\left|+\wp_{2} E_{2}\right| a\right\rangle\langle c|+\text { H.c. }
$$

describes the interaction between radiation and atomic system in dipole approximation; $\wp_{1,2}$ are the dipole moments of the atomic transitions, $E_{1,2}=\mathcal{E}_{1,2} \cos \left[k_{1,2} z-\nu_{1,2} t\right]$ are the fields, and $\mathcal{E}_{1,2}$ are the amplitudes of the fields. Then the atomic states $|a, p\rangle,|b, p\rangle,|c, p\rangle$ now include momentum states $|p\rangle$ that describe the center-of-mass motion. The momentum states are the eigenstates of operators of the momentum and the kinetic energy

$$
\hat{p}|p\rangle=p|p\rangle, \quad \frac{\hat{p}^{2}}{2 M}|p\rangle=\frac{p^{2}}{2 M}|p\rangle,
$$

and they can be written as

$$
|p\rangle=e^{-i p z / \hbar}|0\rangle \text {. }
$$

The state vector $|\Psi\rangle=A\left|a, p^{\prime}\right\rangle+B|b, p\rangle+C\left|c, p^{\prime \prime}\right\rangle$ obeys the Schrödinger equation

$$
i \hbar|\dot{\Psi}\rangle=\hat{H}|\Psi\rangle,
$$

which can be written as the set of the following equations:

$$
\begin{gathered}
i \hbar \dot{A}=\left(\hbar \omega_{a}+\frac{p_{z}^{\prime 2}}{2 M}\right) A+\wp E_{1} B\left\langle p^{\prime} \mid p\right\rangle+\wp E_{2} C\left\langle p^{\prime} \mid p^{\prime \prime}\right\rangle, \\
i \hbar \dot{B}=\frac{p^{2}}{2 M} B+\wp E_{1} A\left\langle p \mid p^{\prime}\right\rangle, \\
i \hbar \dot{C}=\left(\hbar \omega_{c}+\frac{p^{\prime \prime 2}}{2 M}\right) C+\wp E_{2} A\left\langle p^{\prime \prime} \mid p^{\prime}\right\rangle
\end{gathered}
$$

Introducing

$$
\begin{gathered}
A=a e^{-i \omega_{a} t-i\left(p^{\prime 2} / 2 \hbar M\right) t}, \\
B=b e^{-i\left(p^{2} / 2 \hbar M\right) t}, \\
C=c e^{-i \omega_{c} t-i\left(p^{\prime \prime 2} / 2 \hbar M\right) t},
\end{gathered}
$$

Eqs. (A6)-(A8) become

$$
\begin{gathered}
i \dot{a}=\frac{\wp \mathcal{E}_{1}}{2 \hbar} b e^{i \Phi_{1}}+\frac{\wp \mathcal{E}_{2}}{2 \hbar} c e^{i \Phi_{2},}, \\
i \dot{b}=\frac{\wp \mathcal{E}_{1}^{*}}{2 \hbar} a e^{-i \Phi_{1}}, \\
i \dot{c}=\frac{\wp \mathcal{E}_{2}^{*}}{2 \hbar} a e^{-i \Phi_{2}},
\end{gathered}
$$

where the phases $\Phi_{1,2}$ are defined as 


$$
\begin{gathered}
\Phi_{1}=i k_{1} z-i\left(\nu_{1}-\omega_{a b}\right) t-i \frac{\left(p-p^{\prime}\right) z}{\hbar}+i \frac{p^{\prime 2}-p_{z}^{2}}{2 M} t, \\
\Phi_{2}=i k_{2} z+-i\left(\nu_{2}-\omega_{a b}+\omega_{c b}\right) t+i \frac{\left(p^{\prime \prime}-p^{\prime}\right) z}{\hbar}+i \frac{p^{\prime 2}-p^{\prime 2}}{2 M} t .
\end{gathered}
$$

Using the conservation of linear momentum gives us

$$
p^{\prime}=p+\hbar k, \quad p^{\prime \prime}=p+\hbar\left(k_{1}-k_{2}\right) .
$$

Then Eqs. (A12)-(A14) are

$$
\begin{gathered}
i \dot{a}=\frac{\wp \mathcal{E}_{1}}{2 \hbar} b e^{i \Delta_{1} t}+\frac{\wp \mathcal{E}_{2}}{2 \hbar} c e^{i \Delta_{2} t}, \\
i \dot{b}=\frac{\wp \mathcal{E}_{1}^{*}}{2 \hbar} a e^{-i \Delta_{1} t}, \\
i \dot{c}=\frac{\wp \mathcal{E}_{2}^{*}}{2 \hbar} a e^{-i \Delta_{2} t},
\end{gathered}
$$

where we introduce the detunings

$$
\begin{gathered}
\Delta_{1}=\omega_{a b}-\nu_{1}+\frac{k_{1} p}{M}+\frac{\hbar k_{1}^{2}}{2 M}, \\
\Delta_{2}=\omega_{a c}-\nu_{2}+\frac{k_{2} p}{M}+\frac{\hbar\left[k_{1}^{2}-\left(k_{1}-k_{2}\right)^{2}\right]}{2 M},
\end{gathered}
$$

and also $\omega_{a c}=\omega_{a b}-\omega_{c b}$. For an optical transition $k$ $\simeq 10^{5} \mathrm{~cm}^{-1}$, a thermal velocity $v_{T} \simeq 10^{4} \mathrm{~cm} / \mathrm{s}$, thus $p \gg \hbar k$, and the recoil terms can be neglected resulting in the following detunings taking into account the Doppler shifts:

$$
\Delta_{1}=\omega_{a b}-\nu_{1}+k_{1} \frac{p}{M}, \quad \Delta_{2}=\omega_{a c}-\nu_{2}+k_{2} \frac{p}{M} .
$$

Now, let us note here that there are simple arguments for the case $p \gg \hbar k$. One is that if we change a reference frame to the moving one with the atom, then the atom is at rest and the results can be applied but we need to transfer the frequency and the wave number by the transformation

$$
k^{\prime}=\frac{k+\frac{v}{c^{2}} \nu}{\sqrt{1-\frac{v^{2}}{c^{2}}}},
$$

$$
\nu^{\prime}=\frac{\nu+k v}{\sqrt{1-\frac{v^{2}}{c^{2}}}} .
$$

For the case of $v \ll c$,

$$
k^{\prime}=k,
$$

$$
\nu^{\prime}=\nu+k v .
$$

The second simplified approach, which is also frequently used, is related to a fact that the atom is moving with a constant velocity, and its position is given by $z=v t$; then this motion modifies the phase of a field as

$$
\cos (k z-\nu t)=\cos [(\nu-k v) t] .
$$

These two simplified consideration gives us exactly the same results coinciding with the regular approach above. The benefit of the regular approach (see also similar approach in [32]) is that we can estimate the neglected terms to understand the limits when the approximation works well.

\section{APPENDIX B: ELEMENTS OF THE MATRIX OF DIPOLE MOMENT}

The electric dipole elements for the transition of the $\mathrm{Rb}$ $D_{1}$ line can be calculated using the Wigner-Eckart theorem [51]. The irreducible components of the dipole moment are the following:

$$
\wp_{0}=e z, \quad \wp_{+1}=e \frac{x+i y}{\sqrt{2}}, \quad \wp_{-1}=e \frac{x-i y}{\sqrt{2}} .
$$

The atomic states are characterized by the electron angular momentum $J$, the nuclear angular momentum $I$, the total angular momentum $F$, and its projection $m$. In this basis, the matrix elements of electric dipole $\wp_{q}$ are given by

$$
\left\langle\left.\alpha^{\prime} I J^{\prime} F^{\prime} m^{\prime}\right|_{\wp_{q}} \mid \alpha I J F m\right\rangle=C_{1 q, F m}^{F^{\prime} m^{\prime}}\left\langle\alpha^{\prime} I J^{\prime} F^{\prime}\left\|_{\wp}\right\| \alpha I J F\right\rangle,
$$

where $\alpha$ is the set of other parameters that characterized the atomic states, $C_{1 q, F m}^{F^{\prime} m^{\prime}}$ 's are the Clebsch-Gordan factors [51], and $\left\langle\alpha^{\prime} I J^{\prime} F^{\prime}\left\|_{\wp}\right\| \alpha I J F\right\rangle$ is the reduced matrix element of the dipole moment.
[1] E. Arimondo, in Progress in Optics, edited by E. Wolf (Elsevier Science, Amsterdam, 1996), Vol. XXXV, p. 257.

[2] M. O. Scully and M. S. Zubairy, Quantum Optics (Cambridge University Press, Cambridge, England, 1997).

[3] S. E. Harris, Phys. Today 50(7), 36 (1997).
[4] J. P. Marangos, J. Mod. Opt. 45, 471 (1998).

[5] M. Fleischhauer, A. Imamoglu, and J. P. Marangos, Rev. Mod. Phys. 77, 633 (2005).

[6] S. E. Harris, Phys. Rev. Lett. 85, 4032 (2000).

[7] A. B. Matsko, Y. V. Rostovtsev, H. Z. Cummins, and M. O. 
Scully, Phys. Rev. Lett. 84, 5752 (2000).

[8] A. B. Matsko, Y. V. Rostovtsev, M. Fleischhauer, and M. O. Scully, Phys. Rev. Lett. 86, 2006 (2001).

[9] Y. V. Rostovtsev, A. B. Matsko, R. M. Shelby, and M. O. Scully, Opt. Spectrosc. 91, 490 (2001).

[10] Y. V. Rostovtsev, Z. E. Sariyanni, and M. O. Scully, Phys. Rev. Lett. 97, 113001 (2006).

[11] Y. V. Rostovtsev, Z. E. Sariyanni, and M. O. Scully, Proc. SPIE 6482, 64820T (2007).

[12] L. V. Hau, S. E. Harris, Z. Dutton, and C. H. Behroozi, Nature (London) 397, 594 (1999).

[13] M. M. Kash, V. A. Sautenkov, A. S. Zibrov, L. Hollberg, G. R. Welch, M. D. Lukin, Y. Rostovtsev, E. S. Fry, and M. O. Scully, Phys. Rev. Lett. 82, 5229 (1999).

[14] D. Budker, D. F. Kimball, S. M. Rochester, and V. V. Yashchuk, Phys. Rev. Lett. 83, 1767 (1999).

[15] E. E. Mikhailov, V. A. Sautenkov, Y. V. Rostovtsev, and G. R. Welch, J. Opt. Soc. Am. B 21, 425 (2004).

[16] R. Coussement, Y. Rostovtsev, J. Odeurs, G. Neyens, P. Mandel, G. Kozyreff, R. Shakhmuratov, and O. Kocharovskaya, Phys. Rev. Lett. 89, 107601 (2002).

[17] A. B. Matsko, O. Kocharovskaya, Y. Rostovtsev, G. R. Welch, A. S. Zibrov, and M. O. Scully, Adv. At., Mol., Opt. Phys. 46, 191 (2001).

[18] A. V. Turukhin, V. S. Sudarshanam, M. S. Shahriar, J. A. Musser, B. S. Ham, and P. R. Hemmer, Phys. Rev. Lett. 88, 023602 (2001).

[19] C. Y. Ye, A. S. Zibrov, Yu. V. Rostovtsev, and M. O. Scully, Phys. Rev. A 65, 043805 (2002).

[20] V. A. Sautenkov, Y. V. Rostovtsev, H. Chen, P. Hsu, G. S. Agarwal, and M. O. Scully, Phys. Rev. Lett. 94, 233601 (2005).

[21] C. Y. Ye, V. A. Sautenkov, Y. V. Rostovtsev, and M. O. Scully, Opt. Lett. 28, 2213 (2003).

[22] H. Xiong, M. O. Scully, and M. S. Zubairy, Phys. Rev. Lett. 94, 023601 (2005).

[23] M. O. Scully, Phys. Rev. Lett. 55, 2802 (1985); M. O. Scully and M. S. Zubairy, Phys. Rev. A 35, 752 (1987).

[24] W. Schleich, M. O. Scully, and H.-G. von Garssen, Phys. Rev. A 37, 3010 (1988); W. Schleich and M. O. Scully, ibid. 37, 1261 (1988).

[25] S. E. Harris, G. Y. Yin, M. Jain, H. Xia, and A. J. Merriam, Philos. Trans. R. Soc. London, Ser. A 355, 2291 (1997).

[26] A. J. Merriam, S. J. Sharpe, M. Shverdin, D. Manuszak, G. Y. Yin, and S. E. Harris, Phys. Rev. Lett. 84, 5308 (2000).

[27] M. O. Scully, G. W. Kattawar, P. R. Lucht, T. Opatrny, H. Pilloff, A. Rebane, A. V. Sokolov, and M. S. Zubairy, Proc. Natl. Acad. Sci. U.S.A. 99, 10994 (2002).

[28] Z. E. Sariyanni and Y. Rostovtsev, J. Mod. Opt. 51, 2637 (2004).

[29] G. Beadie et al., Opt. Commun. 244, 423 (2005).

[30] D. Pestov et al., Science 316, 265 (2007).

[31] W. Maichen, F. Renzoni, I. Mazets, E. Korsunsky, and L. Windholz, Phys. Rev. A 53, 3444 (1996); E. A. Korsunsky, N. Leinfellner, A. Huss, S. Baluschev, and L. Windholz, ibid. 59, 2302 (1999); D. Kosachiov, B. Matisov, and Yu. Rozhdestvensky, Opt. Commun. 85, 209 (1991); A. F. Huss, E. A. Korsunsky, and L. Windholz, J. Mod. Opt. 49, 141 (2002); E. A. Korsunsky and D. V. Kosachiov, Phys. Rev. A 60, 4996
(1999); Z. Li, L.-P. Deng, L.-S. Xen, and K. Wang, Eur. Phys. J. D 40, 147 (2006).

[32] G. Morigi, S. Franke-Arnold, and G.-L. Oppo, Phys. Rev. A 66, 053409 (2002); S. Kajari-Schroder, G. Morigi, S. FrankeArnold, and G.-L. Oppo, ibid. 75, 013816 (2007).

[33] E. A. Wilson, N. B. Manson, C. Wei, and L. Yang, Phys. Rev. A 72, 063813 (2005); D. V. Kosachiov and E. A. Korsunsky, Eur. Phys. J. D 11, 457 (2000).

[34] M. S. Shahriar and P. R. Hemmer, Phys. Rev. Lett. 65, 1865 (1990).

[35] A. S. Zibrov, A. B. Matsko, and M. O. Scully, Phys. Rev. Lett. 89, 103601 (2002); A. S. Zibrov, A. A. Zhukov, V. P. Yakovlev, and V. L. Velichansky, JETP Lett. 83, 136 (2006).

[36] S. F. Yelin, V. A. Sautenkov, M. M. Kash, G. R. Welch, and M. D. Lukin, Phys. Rev. A 68, 063801 (2003).

[37] K. Yamamoto, K. Ichimura, and N. Gemma, Phys. Rev. A 58, 2460 (1998).

[38] T. Abi-Salloum, J. P. Davis, C. Lehman, E. Elliott, and F. A. Narducci, J. Mod. Opt. 54, 2459 (2007).

[39] M. V. Pack, R. M. Camacho, and J. C. Howell, Phys. Rev. A 74, 013812 (2006).

[40] V. A. Sautenkov, H. Li, Y. V. Rostovtsev, G. R. Welch, J. P. Davis, F. A. Narducci, and M. O. Scully, J. Mod. Opt. 55, 3093 (2008); 56, 975 (2009).

[41] G. S. Agarwal and T. N. Dey, Phys. Rev. A 68, 063816 (2003).

[42] Y. Rostovtsev, O. Kocharovskaya, and M. O. Scully, J. Mod. Opt. 49, 2637 (2002); O. Kocharovskaya, Y. Rostovtsev, and M. O. Scully, Phys. Rev. Lett. 86, 628 (2001).

[43] N. S. Ginsberg, S. R. Garner, and L. V. Hau, Nature (London) 445, 623 (2007); C. Liu et al., ibid. 409, 490 (2001); D. F. Phillips, A. Fleischhauer, A. Mair, R. L. Walsworth, and M. D. Lukin, Phys. Rev. Lett. 86, 783 (2001).

[44] A. S. Zibrov, A. B. Matsko, O. Kocharovskaya, Y. V. Rostovtsev, G. R. Welch, and M. O. Scully, Phys. Rev. Lett. 88, 103601 (2002).

[45] I. Novikova et al., Phys. Rev. Lett. 98, 243602 (2007); I. Novikova, D. F. Phillips, and R. L. Walsworth, ibid. 99, 173604 (2007); M. Klein, Y. Xiao, A. V. Gorshkov, M. Hohensee, C. D. Leung, M. R. Browning, D. F. Phillips, I. Novikova, and R. L. Walsworth, Proc. SPIE 6904, 69040C (2008).

[46] A. V. Gorshkov, A. Andre, M. D. Lukin, and A. S. Sorensen, Phys. Rev. A 76, 033804 (2007); 76, 033805 (2007); 76, 033806 (2007).

[47] Q. Sun, Y. V. Rostovtsev, J. P. Dowling, M. O. Scully, and M. S. Zubairy, Phys. Rev. A 72, 031802(R) (2005).

[48] H. Lee, Y. Rostovtsev, C. J. Bednar, and A. Javan, Appl. Phys. B: Lasers Opt. 76, 33 (2003); A. Javan, O. Kocharovskaya, H. Lee, and M. O. Scully, Phys. Rev. A 66, 013805 (2002); Y. Rostovtsev, I. Protsenko, H. Lee, and A. Javan, J. Mod. Opt. 49, 2501 (2002).

[49] Wolfgang Demtroder, Laser Spectroscopy: Basic Concepts and Instrumentation (Springer, Berlin, 2003).

[50] Murray Sargent III, Marlan O. Scully, and Willis E. Lamb, Laser Physics (Addison-Wesley Publishing Co., Advanced Book Program, Reading, MA, 1974); Pierre Meystre and Murray Sargent III, Elements of Quantum Optics (Springer, Berlin, 2007).

[51] R. N. Zare, Angular Momentum (Wiley, New York, 1988). 